\title{
Guest Editorial ARC 2014
}

The 10th International Symposium on Applied Reconfigurable Computing (ARC 2014) was held in Vilamoura, Algarve, Portugal, April 14-16, 2014. The symposium was hosted by the University of Porto, Faculty of Engineering. ARC 2014 brought together researchers and practitioners of reconfigurable computing, with an emphasis on practical applications of this promising technology. The symposium had three invited international speakers who expressed their views on the future of reconfigurable technology. The accepted papers were included in the proceeding published by Springer Lecture Notes in Computer Science (LNCS), vol. 8405.

It is our pleasure to present in this special ACM TRETS section improved and extended versions of three articles previously accepted and presented at ARC 2014 . The articles included in this special section, and which research contributions and highlights we present below, have been selected out of the 33 papers presented at the conference and cover various topics, namely reconfiguration techniques, over-clocking, and image coding in the context of embedded computing.

The article entitled "On the Impact of Replacing Low-Speed Configuration Buses on Field Programmable Gate Arrays (FPGAs) with the Chip's Internal Configuration Infrastructure," by Karel Heyse, Jente Basteleus, Brahim Al Farisi, Dirk Stroobandt, Oliver Kadlcek, and Oliver Pell, proposes a methodology for accessing block memories and registers on FPGAs of the Maxeler HPC platform using partial reconfiguration instead of using the low-speed memory bus. This approach has been evaluated with one financial and two geoscience applications. Results in terms of improved clock frequency, read and write speeds of the mapped memories, FPGA resource utilization, and compilation time are presented. In addition, suggestions for improvements in the Xilinx infrastructure and tools are given that would make the proposed methodology more widely applicable.

The article entitled "Over-Clocking KLT Designs on FPGAs Under Process, Voltage and Temperature Variation," by Rui Policarpo Duarte and Christos-Savvas Bouganis, presents a novel methodology to optimize designs for the widely used linear projection known as the Karhunen-Loeve Transformation (KLT) algorithm. The authors characterize the arithmetic units in the datapath given different operating conditions and process, voltage, and temperature variations. The results show that in three real-world use cases the methodology results in better designs compared to typical implementations of the KLT algorithm.

The article entitled "Toward a Fast FPGA Implementation of Heap-Based Priority Queue for Image Coding Using Parallel Index-Aware Tree," by Yuhui Bai, Syed Ahmed, and Bertrand Granado, presents a System-on-Chip (SoC) FPGA implementation of a wavelet-based image encoder known as the Öktem image coder. The approach includes an application-specific architecture for the most computationally intensive block of the image encoder, an Adaptive Scanning of Wavelet Data module. It employs an efficient heap-based priority queue based on an index-aware heap structure with parallel tree access, and it uses the on-chip configurable memory capabilities of FPGAs. The architecture, validated as an accelerator in a Nios II processor-based SoC implemented using an Altera's Stratix IV FPGA, provides a $45 \times$ speedup at $150 \mathrm{MHz}$ over an embedded ARM Cortex-A9 processor at $666 \mathrm{MHz}$.

(c) 2015 ACM 1936-7406/2015/11-ART5 $\$ 15.00$

DOI: http://dx.doi.org/10.1145/2831431

ACM Transactions on Reconfigurable Technology and Systems, Vol. 9, No. 1, Article 5, Publication date: November 2015. 
As guest editors, we would like to acknowledge all authors of the articles of this section for the high quality of their work as well as the anonymous reviewers for their reviewing efforts and suggestions for improving previous versions of these articles.

We hope you enjoy reading the papers included in this special section and that they serve as useful sources of research inspiration.

Diana Goehringer

Ruhr-University Bochum (RUB), Bochum, Germany (diana.goehringer@rub.de)

Marco D. Santambrogio Politecnico di Milano, Milan, Italy (marco.santambrogio@polimi.it)

João M. P. Cardoso

Universidade do Porto, Porto, Portugal (jmpc@acm.org)

Koen Bertels

TUDelft, Delft, The Netherlands (k.l.m.bertels@tudelft.nl)

Guest Editors 